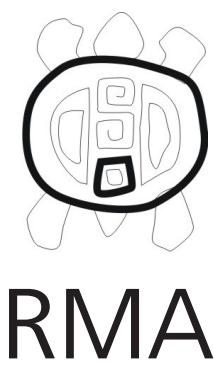

Dossier

\title{
Etnografía Argentina: La cátedra libre de Monseñor Pablo Cabrera (1925)
}

\author{
Mariela Eleonora Zabala
}

Museo de Antropología, Facultad de Filosofía y Humanidades, Universidad Nacional de Córdoba - Becaria Secyt (UNC).E-mail: marielaeleonora@gmail.com

\begin{abstract}
Resumen
La Reforma Universitaria de la Universidad Nacional de Córdoba (UNC) en 1918, posibilitó la inserción académica de la antropología en el área central argentina, mediante dos iniciativas de los estudiantes. Una fueron los cursos y docencia libre de carácter extracurricular, para alumnos de todas las carreras. Otra fueron los concursos docentes, destinados a excluir de la autoridad, la administración y el cuerpo docente a los católicos y conservadores, y beneficiar a los profesores "más capaces y dignos" en nombre de "la idoneidad", "la justicia" y "la libertad".

Este artículo examina una derivación directa de estas iniciativas que parece contradecir sus propósitos: la emergencia del primer curso libre de Etnografía Argentina en la UNC en 1925. Dicho espacio curricular fue propuesto y estuvo a cargo de Monseñor Pablo Cabrera (1857-1936), sacerdote de la diócesis de Córdoba, miembro de la Academia Nacional de Ciencias, director del Museo Histórico Provincial, y prolífero autor de libros y artículos publicados en la Revista de la Universidad y el diario Los Principios. ¿Cómo fue posible que un intelectual de la Iglesia encabezara semejante innovación en un contexto anticlerical? ¿Cómo incidió su adscripción confesional en una institución laica, y cómo incidió su obra universitaria en su vertiente clerical? ¿Cómo convivieron estas dos líneas en una misma persona, precursor de la antropología cordobesa?
\end{abstract}

Palabras Claves: Reforma universitaria; enseñanza; Etnología argentina; Monseñor Pablo Cabrera.

Argentine Ethnography: Monsignor Pablo Cabrera's free lecture (1925)

\begin{abstract}
The groundbreaking Reform that took place at the National University of Córdoba (Universidad Nacional de Córdoba, UNC) in 1918, paved the way to the academic start of anthropology in Central Argentina. This new presence was fostered by two initiatives of the students' sector: extra-curriculum or "free" courses, and public contests to professors' positions. The latter was meant to exclude the Catholic and conservative personnel from the leading and teaching positions and from the administration, and to ensure access of the "most capable and dignified" professors to the University, in the name of capacity, justice and freedom.

This paper explores a direct effect of such initiatives which seem to oppose such goals: the launching of the first free course of Argentine Ethnography by Prior (Monseñor) Pablo Cabrera (1857-1936) in 1925. Cabrera, its main professor, was a member of the Catholic Church of Córdoba and of the National Academy of Sciences, the director of the Provincial Historical Museum, and the author of myriad books and articles on Revista de la Universidad and the newspaper Los Principios. ¿Why and how a man of the Church launched this innovation in the anti-clerical context of the University Reform? How did his confessional adscription affect Cabrera's work in a secular institution and conversely how did his academic stance impinge on his priestly activities? In sum, how could these two realms coexist within the single mind of a man acknowledged as the predecessor of Cordobesa anthropology?
\end{abstract}

Keywords: University reform; teaching; Anthropology; Pablo Cabrera; Córdoba; Argentina.

En 1918 una revuelta estudiantil en la ciudad de Córdoba dio lugar a lo que se conoce como Reforma Universitaria. Este fue un hecho fundamental en el proceso de secularización del sistema educativo que enfrentaba a la Iglesia con los movimientos intelectuales liberales de la época. Fue éste el eslabón final de la lucha entre conservadores y liberales, y entre generaciones, en una Córdoba que se pretendía modernizada y por eso libre de la fuerte tradición clerical e hispana que había dado origen, precisamente, al primer sistema educativo del territorio que luego fue la Argentina (Aguiar 2008).

La reforma nació de las protestas contra el cierre del internado del Hospital de Clínicas, hecho que los estudiantes aprovecharon para pedir cambios en los estatutos y en los planes de estudios. En el Manifiesto 
Liminar la reforma quedaba definida como iconoclasta, liberal y anticlerical. Los jóvenes que formaron parte del movimiento vieron la posibilidad de construir un discurso contra-hegemónico para disputar la primacía política y cultural al público católico que dominaba prácticamente todas las instituciones de la provincia y, también, de la educación superior (Aguiar 2008; Requena 2008).

Así, para Vidal (2005:1)“la reforma fue un grito de modernidad lanzado por los estudiantes cordobeses, con importantes adhesiones de diversas instituciones y figuras intelectuales y políticas del país e incluso del extranjero".

Este hito en la historia de la universidad tuvo una gran importancia para la antropología argentina en la región central, mediante dos iniciativas de los estudiantes. Una fue la creación de cursos y docencia libre a pedido de los estudiantes en el movimiento reformista, y que se efectivizaron durante la intervención nacional universitaria de Nicolás Matienzo (1918). Estos cursos eran de carácter extracurricular, y estaban destinados a los alumnos de todas las carreras, de manera que lo que se enseñaba en ellos se propagaba a través de las carreras. En este espacio y según la resolución emitida por el Honorable Consejo Superior de la universidad en la sesión del 28 de mayo de 1925, Cabrera dictaría el curso de Investigaciones sobre Etnografía Argentina, fue a pedido de quién que fuera él? ¿De los estudiantes? ¿Ellos eligieron el tema o tema y profesor? Entre tanto el Dr. Ariosto Licurssi ${ }^{1}$ fue convocado a dictar Antropología.

Monseñor Pablo Cabrera (1857-1936) estudió en la Universidad Nacional de Córdoba en tiempos en que no había diferencia entre quienes aspiraban al sacerdocio y quienes aspiraban a ser abogados. Esto fue así hasta 1881 cuando se creó la Licenciatura en Teología, lo cual generó la división entre la universidad y la Iglesia para la formación de los sacerdotes porque, tanto el Rector como el Obispo de la Diócesis de Córdoba se autoasignaban la prerrogativa de nombrar a sus respectivos docentes. El Obispo se basaba en leyes canónicas y el Rector en leyes nacionales. Este diferendo fue el primero de una progresiva secesión entre dos instituciones que, en tiempos de la colonia y bajo la orden jesuítica, habían compartido una misma dirección y transmisión de los estudios superiores.

Cabrera se ordenó como sacerdote en la Diócesis de Cuyo pero ejerció sus tareas pastorales en la de Córdoba, como capellán del Colegio de las Hermanas Esclavas del Corazón de Jesús (1884-1896), de la Iglesia de Nuestra Señora del Pilar (1895-1929) y como miembro de la Comisión de Liturgia (1905). Sin embargo, su desempeño no fue sólo

\footnotetext{
1 Licurssi se recibió de médico en 1921 en la Universidad Nacional de Córdoba. Docente de distintas carreras y materias como Zoología, Medicina Legal y Toxicología. También fue Director del Instituto de Criminología de Córdoba, entre otros cargos docentes y de funcionario provincial.
}

eclesiástico. También fue miembro del Ateneo de Córdoba (1894), de la Academia Nacional de Ciencias (1915), y director del Museo Histórico Provincial (1919). Además, en 1924 fundó la Junta de Estudios Históricos que en 1928 se transformó en Junta de Historia y Numismática Americana. En el ámbito nacional fue designado socio activo de la Sociedad Científica Argentina (1934) y en el internacional fue miembro de la prestigiosa Sociedad de Americanistas de París (1927). Fue un prolífero autor de libros y artículos publicados en la Revista de la Universidad y el diario Los Principios. ¿Cómo fue posible que un intelectual de la Iglesia encabezara semejante innovación en un contexto por principios anticlerical? ¿Cómo incidió su adscripción confesional en una institución laica y racional, y cómo incidió su obra universitaria en su vertiente clerical? ¿Cómo convivieron estas dos líneas en una misma persona, precursora de la antropología de la capital del interior argentino?

\section{La cátedra de Etnografía Argentina}

Entre las iniciativas reformistas tendientes a transformar planteles docentes y ofertas de formación se contaban los concursos y las cátedras libres. Los concursos docentes estaban destinados a excluir de la autoridad, la administración y el cuerpo docente a los católicosreligiosos o laicos - y a los conservadores, beneficiando a los profesores "más capaces y dignos" en pos de "la idoneidad", "la justicia" y "la libertad". Además, y según el Acta del Consejo Superior del 19 de abril de 1918, se establecía "conceder permiso para dictar cursos libres sobre materias que se relacionan con su enseñanza, reglamentado la docencia libre en forma que puedan darse, mediante ella cursos similares a los oficiales con derecho para los estudiantes de seguirlos en lugar de estos $^{2 \prime}$. Estos cursos abrían la posibilidad de incorporar nuevos docentes a la enseñanza universitaria y nuevos campos de estudio, derramándose por diversas carreras, ya que no eran exclusivas. Sus profesores podían ser suplentes, diplomados universitarios, nacionales o extranjeros o personas competentes reconocidas. El docente hacía la propuesta, y el Consejo Superior debía aprobarla.

De este espacio hizo uso Cabrera para incorporarse a la docencia universitaria y, simultáneamente, incorporó al mundo universitario cordobés los estudios sobre etnografía, un campo del saber que estaba en formación en la Argentina. La Universidad de Buenos Aires y la Universidad de La Plata ya contaban con cátedras de Arqueología y Antropología pero muy lejos estaban de las licenciaturas que se crearon en 1958 y 1957 respectivamente.

De dicho curso sólo sabemos que el Consejo Superior de la Universidad de Córdoba, en sesión del 17 de junio de 1925, por proposición de la Comisión de Enseñanza,

\footnotetext{
${ }^{2}$ AGHUNC, Actas del Consejo Superior año 1918.
} 
designó por lo que quedaba del año al "Dr. Pablo Cabrera" para dictar en calidad de curso libre un ciclo de conferencias entre 10 como mínimo y 25 como máximo. Su temática giraría en torno a las "Investigaciones sobre Etnografía Argentina" ${ }^{\prime 3}$ sin más especificidad. Pese a la falta de información, y ya para 1925 tenemos algunos elementos para imaginar cuál sería su contenido, por lo que debemos echar una mirada a sus antecedentes. Estos seguramente sustentaron la idoneidad del profesor y también su posibilidad de ser integrado sin ser denostado por los estudiantes que pedían la expulsión de los miembros de la Iglesia de los claustros universitarios.

\section{La trayectoria de Cabrera como sacerdote en la Diócesis de Córdoba}

Cabrera era, como suele definir a sus miembros la máxima institución Católica, "un hombre de la Iglesia". Ya en los años 20 había tenido un gran desempeño como "publicista" y orador en dicha institución pero sin ocupar un lugar de docencia en la formación de sacerdotes, ni en el Museo del Seminario (creado en 1918), ni en el Archivo del Obispado.

Su primera publicación fue en abril del significativo año de 1883, en la provincia de Mendoza, y versó sobre un tema eclesial. Por consiguiente, y previo a su publicación el manuscrito debía someterse a la aprobación de las autoridades eclesiásticas, cosa que se especifica en la cubierta del volumen. Fundamentos de la religión estaba dedicado a su tío, el presbítero Eleuterio Mercado. Su objetivo era "Propagar los principios religiosos y poner al común alcance, el conocimiento de las pruebas y de los fundamentos de nuestra religión". Sus destinatarios eran no tanto los religiosos, sino los laicos y especialmente aquéllos que no contaban con estudios superiores. En este libro de divulgación de la fe, con claridad y contundencia, Cabrera afirmaba que las "verdades naturales de la razón" deben someterse a las "verdades divinas reveladas". Seguidamente, se volcaba a analizar "El materialismo y el origen del hombre", donde rechazaba "El sistema inventado por Charles Darwin" (1809-1882) y su teoría de la evolución, por considerarlos una "Ridiculez y absurdidad". Seguidamente explicaba el origen divino y humano de los libros bíblicos; el vínculo basado en el derecho natural y divino entre el Estado y la Iglesia; y finalmente el análisis del "Syllabus" (lat. "listado de los errores") o enumeración de los principales errores que atravesaba el mundo moderno ${ }^{4}$, tales como "el panteísmo 5", "el naturalismo", "el racionalismo absoluto y

\footnotetext{
3 AAC, Caja 1. Monseñor Pablo Cabrera.

4 Bajo el papado de Pío IX se publicó el Syllabus donde se estipulan y condenan las principales libertades modernas. Este documento se dio a conocer el 8 de diciembre de 1864 aunque se había comenzado a tratar en 1849, y por distintos motivos se fue dilatando su redacción final.

${ }^{5}$ Doctrina de quienes creen que la totalidad del universo es el único Dios (Diccionario Religioso de Henzo Lafuente).
}

moderado", "el indiferentismo", "el latitudinarismo6", "el socialismo", "el comunismo", "las sociedades secretas" y "el liberalismo". También condenaba "el vicio y la barbarie" disfrazados bajo el nombre de "La civilización y del progreso", invirtiendo de manera interesante la antinomia planteada por otro sanjuanino pero esta vez (demasiado) laico, Domingo F.Sarmiento cuanto dicotomizaba entre "civilización o barbarie".

La segunda publicación, también a cargo de la Iglesia y en 1883, era Cuestiones de Actualidad. Los liberales de Allende ó sea el Si y el No acerca de la enseñanza religiosa en las escuelas populares. Su casa editora era mendocina, la Tipografía "Bazar Madrileño". Motivaba el texto "El debate caluroso en las Cámaras nacionales argentinas de la importantísima y trascendental cuestión, de si se debe o no enseñar religión en las escuelas de la República". En la introducción contraponía a los defensores del "Liberalismo" que abogaban por la "Escuela laica, la escuela sin Dios", y los "Clérigos" que pedían la continuidad de "Una escuela cristiana, la escuela con Dios". A los liberales los acusaba de responder a los intereses de la "Franco masonería" mientras que a los clérigos les reconocía la búsqueda de la "Satisfacción de las aspiraciones de la inmensa mayoría de argentinos". "De su resolución (de tal antinomia) depende en gran parte, el porvenir de la civilización, de la libertad y del bienestar de nuestra patria. O hemos de retrogradar al paganismo", tras de lo cual concluía que "Quiera Dios que triunfe la buena causa, y que prevalezcan los buenos principios, que con tanto denuedo y lealtad defienden los diputados católicos, mirando por el bien de la familia y de la sociedad". Si la religión lograba mantenerse en la formación de niños y jóvenes, como aspiraba el presbítero Cabrera, el poder de la Iglesia no se pondría en riesgo ni tampoco la verdad revelada que podría coexistir junto a la de la ciencia moderna. Pero esa pervivencia era altamente improbable, como lo había mostrado, el año anterior, la ley del Congreso Nacional que establecía el Registro Civil y la consagración del matrimonio por la ley nacional y no por la ley eclesiástica. El poder de la Iglesia sobre la vida de los argentinos se recortaba a ojos vista bajo el laicismo del presidente Julio A. Roca.

Cuando regresó a la ciudad de Córdoba luego de estas publicaciones, Cabrera fue nombrado cura capellán (1884-1896) del Colegio de las Hermanas Esclavas del Corazón de Jesús, un establecimiento de niñas y sede del noviciado de la congregación, fundado años antes en un "pueblo nuevo" Pueblo General Paz, actual barrio General Paz. Del otro lado del río Suquía estaba la Iglesia de Nuestra Señora del Pilar a las márgenes de la ciudad colonial. Sobre un total de poco más de 20.000 habitantes en dicha parroquia según el presbítero, a partir de los datos que le ofreció la Oficina de Estadísticas de la Provincia, había unos 300 protestantes con dos salones

\footnotetext{
${ }^{6}$ Doctrina que entiende que puede haber salvación fuera de la Iglesia Católica.
} 
(templos), uno dedicado a la primera enseñanza y otro al canto ${ }^{7}$. Cabrera ejercía como párroco en un espacio de frontera con la modernización, con una población nacional y socialmente diversa.

Destacada fue su "oratoria" en el Tedeum ${ }^{8}$ de "acción de gracias" por el pacto de límites entre las Repúblicas de Chile y de Argentina, que tuvo lugar el 24 de agosto de 1902 nada menos que en la Catedral de Buenos Aires. El discurso de Cabrera estuvo dirigido a notabilísimas figuras presentes con sus valiosos títulos: el Presidente de la República Teniente General Julio A. Roca, el Internuncio Apostólico Monseñor Antonio Sabatucci y el Arzobispo Monseñor Mariano A. Espinosa. Esta intervención es sorprendente por la baja investidura de Cabrera, un simple "sacerdote" convocado a ofrecer un sermón en otra diócesis que no era la propia, y además en una circunstancia de gran relevancia para la Iglesia Católica y para ambas Repúblicas; el escenario era, ni mas ni menos, el centro político y eclesiástico de la Argentina: la Catedral.

El inmenso honor y el extraordinario voto de confianza por parte del Obispo de Córdoba que permite a Cabrera ofrecer el sermón del Tedeum, tal vez en su reemplazo por no poder asistir, y por parte del Obispo de Buenos Aires que lo recibió y le cedió "la palabra", son difíciles de imaginar. Y el presbítero no los defraudó, haciendo gala de sus conocimientos sobre los tratados previos de Paz con Chile, sobre historia, filosofía y, por supuesto, teología. En una ocasión de importancia gigantesca, muy similar a una consagración, mostraba Cabrera su erudición en distintos campos del saber acudiendo a autores nacionales e internacionales con claridad y contundencia. A continuación, el 13 de marzo de 1904 Cabrera oró al pie del Monumento del Cristo Redentor en la Cordillera mendocina, luego de conocido el fallo de "Su Majestad Británica" por los límites a $23^{\circ}$ y $26^{\circ}$ de latitud austral (Cabrera 1904). La devoción por el Cristo Redentor obedecía a un pedido del Papa León XIII por la paz y la armonía en el mundo? .

Cabrera desde entonces regresaría a la ciudad de Córdoba, desde una nueva jerarquía consagrada por sus actuaciones en Chile y sobre todo en la Catedral de Buenos Aires, única arquidiócesis de todo el territorio argentino. El presbítero Cabrera rozaba el poder político terrenal y celestial, entre las Sagradas Escrituras y las verdades temporales de los estadistas y hombres de ciencias.

Entonces, ¿cómo fue que con estas publicaciones a favor

\footnotetext{
7 Información declarada por Monseñor Pablo Cabrera ante la Visita Canónica de 1905. Caja 2 Monseñor Pablo Cabrera del AAC.

8 El Tedeum es un acto que se realiza en las ciudades con catedrales donde se reúnen a rezar por la Patria el obispo y las autoridades políticas locales. Siempre se celebran el 25 de mayo pero pueden convocarse para otras ocasiones especiales.

${ }^{9}$ Según la Encíclica Tametsi futura prospicientibus del $1^{\circ}$ de noviembre de 1900, escrita por León XIII.
}

de la enseñanza religiosa y en contra de la teoría de Darwin y los "errores modernos", pudo integrarse en el mundo académico-universitario cordobés?

\section{La trayectoria de Cabrera como especialista en la universidad}

Cabrera tuvo un gran desempeño como "publicista" e investigador hasta que en 1925 se incorporó como docente en la universidad. En 1906 fue designado encargado de la investigación en archivos. Esta tarea le permitió pedir al Rector, en 1907, de que solicitará al gobierno de la Provincia de Córdoba un volumen del Index librovum Biblioteca y Collegü Máximi, Cordubensis Societatis Jesu (1757), entre otras publicaciones pedidas a nivel provincial, nacional como internacional; y ser el encargado de la colección y copia de los documentos relacionados con la historia de la universidad. Intuyo que estas tareas deben haber contribuido para su aparición en 1910 en dos eventos académicos de gran notoriedad: el Congreso Internacional de Americanistas, entre el 17 al 23 de mayo, en la Ciudad de La Plata (Provincia de Buenos Aires) y en representación de la UNC en el Congreso Científico Internacional Americano, del 10 al 25 de julio. Fue en este último que presentó su investigación sobre "Los Lules" en la Comisión de Ciencias Antropológicas coordinada por Florentino Ameghino. En la misma comisión, a pedido del coordinador, presidió la sesión donde Roberto Lehmann Nitsche (1872-1930) presentó su trabajo sobre "El problema indígena. Necesidades de destinar territorios reservados a los indígenas en Patagonia, Tierra del Fuego y Chaco según el proceder de los Estados Unidos de Norte América". Además, intervino en discusiones sobre cuestiones lingüísticas a pedido de Samuel Lafone Quevedo.

A su regreso a Córdoba, Los Lules fue publicado por la Universidad Nacional de Córdoba y un año más tarde ocupaba el cargo de colector de documentos, y en sintonía con la tarea en 1916 ocupó el puesto de "jefe de manuscritos". Más adelante muestro que importancia le otorgaba Enrique Martínez Paz (1882-1952) a esta tarea universitaria.

En el espacio editorial de la universidad fue designado por el Consejo Superior para ser el "secretario redactor" de Anales históricos a partir de 1914 en reemplazo de otro sacerdote, el Reverendo Fray Zenón Bustos y Ferreyra $^{10}$, quién ejercía el cargo desde el año 1899. Según la ordenanza del Consejo Superior de 1891 Anales se publicaría trimestralmente y "cuando la redacción histórica llegase a la época presente, aparecerá sólo una vez al año" (Anales, 1901). Cabrera nunca editó ningún ejemplar de Anales como sí lo hizo Bustos que editó 3 volúmenes sobre el período que los franciscanos gobernaron la universidad (1767-1778, tomo 1; 1778-

\footnotetext{
10 Perteneciente a la orden Franciscana y obispo de la Diócesis de Córdoba entre 1905-1925.
} 
1795, tomo 2; y 1795-1807, tomo 3). Vale señalar que Bustos pertenecía a la orden religiosa de los franciscanos.

En 1914 también la universidad lanzó la Revista institucional, entonces veamos el perfil de la publicación y si Cabrera aportó algún artículo. En el primer tomo de la Revista, su director Martinez Paz, evocaba la existencia de una "universidad antigua" que debió soportar "el peso enorme de la posesión de la verdad" en tiempos en que "la vida debía amoldarse a la verdad y no la verdad a la vida" y se unificaba el saber bajo un "único sistema filosófico". De modo especial quien soportó esta responsabilidad fue la Facultad de Derecho. El modo de aprender esa verdad era a través de "un viejo libro clásico parafraseado con habilidad en el aula" y luego, "confiado a la memoria en los exámenes, aseguraba el éxito y la posesión". En cambio, reflexionaba, la universidad de comienzos del siglo XX se gestaba en el "progreso real del conocimiento que venía de la mano de la ciencia independiente". Esa independencia se había iniciado en las ciencias naturales, lo que no ocurría con las ciencias del hombre. Por eso, esta revista representaba "una tribuna levantada para la dilucidación de los grandes problemas actuales e históricos de nuestra vida". Uno de los grandes problemas a los que hacía referencia eran los "históricos", que estaban "envueltos en la obscuridad de las posiciones, puesto que el conocimiento del pasado, puede ponernos en presencia de la verdadera realidad actual" (Martinez Paz, 1914: 4-7).

Las secciones de la Revista comprendían un apartado donde se publicaban "documentos inéditos, particularmente de interés histórico y universitario", porque los documentos contribuían a "esclarecer la verdad" y a "establecer la exactitud" en desmedro del género literario de la historia más próxima al género novelesco que a la ciencia. Para Martínez Paz la colección y el ordenado de documentos debía dejar de estar en manos de "eruditos y anticuarios" para pasar a manos de "hombres de ciencia". Ese rol fundamental para el desarrollo científico de las ciencias del hombre según Martinez Paz, lo ocupaba Cabrera.

Presbítero ya investido Monseñor, publicó en la Revista cuarenta artículos desde 1914 hasta 1935, meses antes de su fallecimiento en enero de 1936. No publicó en 1919, 1922 y 1923, en algunos años solo uno, y en 1917 publicó cinco artículos. Además Cabrera aparecía como "noticia universitaria", por ejemplo, cuando se informaba del dictado de su Curso en 1925, o cuando la universidad le entregó el título de Doctor Honoris Causa en 1927, o cuando cumplió sus bodas de oro sacerdotales en 1933. Su obituario también ocupó estas páginas, y otra vez para el centenario de su nacimiento.

De los años silenciosos, 1919 era por demás elocuente, pues podía ser consecuencia de la Reforma, pero al año siguiente publicó no sólo en la Revista sino que el rectorado autorizó la inversión de $\$ 500$ pesos nacionales para imprimir su folleto Trejo y su Obra, el cual fue reeditado en la Revista en el mismo año. En verdad el escrito venía a debatir con Antonio Rodríguez del Busto quien en su artículo del diario mitrista La Nación de noviembre del 19 señalaba que el Obispo Trejo y Sanabria no había fundado ni el Colegio de la Compañía de Jesús ni la Universidad. Cabrera entonces empezaba su artículo interpretando el error de Bustos por carecer de información suficiente, de datos y antecedentes que sólo pueden suministrar los documentos conservados en los archivos. Pos reforma universitaria, Así en el órgano más importante de difusión institucional en el período post Reformista, Cabrera podía plantear una discusión de proyección nacional.

\section{Conclusiones}

Es cierto que el diferendo Universidad-Iglesia Católica ha permeado la construcción de un sistema de instrucción laico por parte del moderno Estado nacional. Sin embargo, en Córdoba reviste un interés particular porque la Iglesia fue la creadora del sistema educativo central del primer período colonial, y también de la Universidad, a través de la Compañía de Jesús. Además, Córdoba fue sede del Obispado con el Seminario de formación de sacerdotes también desde el período colonial. Así, referirse a la Universidad y la Iglesia en esta ciudad y en esta provincia es, en cierto período, hablar de una y la misma cosa, lo cual hace prever las enormes consecuencias que tuvo en el ámbito local su progresiva diferenciación.

Parte de ello fue la Reforma Universitaria. Los jóvenes reformistas buscaron romper definitivamente con lo que identificaban como "viejas formas de enseñanza" encarnada, en parte, por los docentes católicos de verdades reveladas más que de verdades racionales y modernas. Sin embargo, fue en el contexto de semejantes cambios que la Etnografía ingresó a la Universidad a través de la cátedra libre dictada por un Monseñor de la Iglesia. Tal respaldo no era inocente: combinaba una trayectoria donde podían convivir una ponencia etnográfica históricolingüística como Los Lules, con la postulación del Nuevo Testamento como única verdad.

Cabrera logró el ingreso al mundo académico-universitario cordobés gracias a un reconocimiento que provino, sobre todo, de "sabios" (como se los llamaba entonces) consumados, y que se performó en dos congresos internacionales (Americanistas y Científico Americano), se hizo público con la publicación de su obra a cargo de la Universidad, la que lo propulsó a la cátedra y al espacio escrito, aunque de otra índole.

Podría pensarse entonces que la presencia de Cabrera en la añosa Universidad de Córdoba era una cuña de la Iglesia Católica en la modernidad cordobesa. Pero esto es sumamente dudoso, porque dicho respaldo y crecimiento de su figura no tuvo correlato con su carrera eclesiástica, 
cuyo último peldaño parece ser su promoción a Monseñor previo a 1910 y a continuación de su participación en el conflicto de límites chileno-argentino ${ }^{11}$. Cabrera era cada vez más reconocido como científico y cada vez menos como intelectual clerical, ya que después de 1910 su afiliación institucional y su comunidad de referencia eran los etnólogos como Lafone Quevedo, y los paleontólogos como Ameghino, miembros de un mundo académico moderno, racional e internacional, reconocido por colegas platenses y, en el ámbito local, por la Universidad y la Academia Nacional de Ciencias a la cual pasó a integrar en 1915. Pero esta pertenencia nunca abandonó a la Iglesia.

Probablemente, Cabrera nunca abdicó de la verdad del dogma, pero sus intereses por la etnología lo derivaron hacia otros ámbitos con otras lógicas de razonamiento y legitimación. Por eso su perfil es el de un académicoreligioso cordobés que en vez de contraponer universidadiglesia, se benefició de la reciprocidad, quizás asimétrica, entre las dos instituciones rectoras de la cultura provincial, que los poderes temporales terminarían ubicando en las antípodas. Es que, como en toda relación de reciprocidad, ésta nunca se consuma total y equilibradamente. Entender el proceso cultural cordobés como un apartamiento definitivo, progresivo y unilineal de la verdad racional, con respecto a los dominios de la Iglesia, es quizás tener una visión bastante simplista de los procesos culturales de esta provincia crucial para el campo intelectual argentino.

\section{Agradecimientos}

Este artículo es el resultado de mi investigación para la tesis de Maestría en Antropología (FFyH-UNC) "Las verdades etnológicas de Monseñor Pablo Cabrera. Una etnografía de archivos en la ciudad de Córdoba", en el marco del Proyecto PICT/R 2006-1728 "Antropología social e histórica del campo antropológico en la Argentina, 1940-1980" (2008-2011), Nodo Córdoba. Le agradezco a Rosana Guber, su directora, la lectura y sugerencias.

\section{Bibliografía}

Aguiar, L. 2008. Ni presión de sectores medios, ni una mera "juvenilia" algo más sobre la Reforma Universitaria. Reseñas de enseñanza de la Historia, Publicación de APEHUN (Asociación de Profesores de Didáctica de la Historia de Universidades Nacionales), $N^{\circ} \mathrm{VI}$, septiembre: 311-342.

\footnotetext{
11 Por otro lado, "Monseñor" es un título honorífico que no significa necesariamente la consagración como Obispo, el grado mayor en la jerarquía eclesiástica.
}

Cabrera, P. 1883. Fundamentos de la Religión. Tipografía "Bazar Madrileño", Mendoza.

Cabrera, P. 1883. Los liberales de Aquende y los liberales de Allende o sea el Si y el No acerca de la enseñanza religiosa en las escuelas populares. Tipografía "Bazar Madrileño", Mendoza.

Cabrera, P. 1904. Cristo en la Cumbre. Compañía Sud. Americana de Billetes de Banco, Buenos Aires.

Cabrera, P. 1910. Ensayos sobre Etnología Argentina. Tomo 1 Los Lules, Universidad Nacional de Córdoba, Córdoba.

Cabrera, P. 1920. Trejo y su obra: a propósito de una publicación adversa a entrambos, B. Cubas, Córdoba.

Martinez Paz, E. 1914. Revista de la Universidad Nacional de Córdoba. Revista de la Universidad, número 1, agosto: 3-8.

Requena, P. 2008. Universidad, política y cultura en la córdoba intersecular o pensar la Reforma Universitaria de 1918 en la mediana duración, 1871-1920. Reseñas de enseñanza de la historia, Publicación de APEHUN (Asociación de Profesores de Didáctica de la Historia de Universidades Nacionales), número VI, septiembre: $287-310$.

Vidal, G. 2005. La modernidad y el espacio público en Argentina. Repensado la Reforma Universitaria de1918. Avances del CESOR, año V, N 5, Rosario.

Vidal, G. 2007. La Reforma Universitaria de 1918 y la Unión Cívica Radical.

Vidal, G. La política y la gente. Estudios sobre modernidad y espacios públicos. Córdoba, 1880-1960, Ferreyra Editor, Córdoba.

\section{Archivos}

Archivo del Arzobispado de Córdoba (AAC)

Archivo General e Histórico de la Universidad Nacional de Córdoba (AGHUNC) 